\title{
General Relativistic Study of Thin Disk with Radial Flow in Kerr - Newman Geometry
}

\author{
Kameshwar Nath Mishra \\ Department of Applied Mathematics, Bhilai Institute of Technology, Durg, India
}

Email address:

knm2@rediffmail.com

\section{To cite this article:}

Kameshwar Nath Mishra. General Relativistic Study of Thin Disk with Radial Flow in Kerr - Newman Geometry. American Journal of Astronomy and Astrophysics. Vol. 5, No. 6, 2017, pp. 57-62. doi: 10.11648/j.ajaa.20170506.11

Received: December 23, 2017; Accepted: January 8, 2018; Published: January 19, 2018

\begin{abstract}
A detailed general relativistic formulation of the thin disk with the radial flow in Kerr - Newman geometry. The effects of rotation through an angular momentum $a$ and charge $Q$ were obtained on pressure, velocity and density for co rotating and counter rotating thin disk.
\end{abstract}

Keywords: Kerr - Newman Black Hole, Thin Disk, Radial Flow

\section{Introduction}

In the study of the charged rotating black hole, the formation of thin disk is one of the most important aspects. We have developed fully general relativistic approach for the dynamics of the thin accretion disk having angular momentum $a$ and charge $Q$ in the Kerr - Newman back ground geometry. When the pressure force is negligible, the disk become a thin, so that confined to the equatorial plane. A solution for thin disk rotating around Kerr - Newman black hole having non zero velocity in the radial direction is obtained. The effect of rotating charge black hole clearly seen by comparing solution with their Kerr and Schwarzschild counterpart.

\section{Mathematical Formulation}

The Kerr - Newman (KN) space-time is an exact solution of the Einstein-Maxwell equations that describes the exterior gravitational and electromagnetic field of a rotating charged source with mass $m$, and angular momentum $a$ and electric charge $Q$. In Boyer -Lindquist coordinate, The $\mathrm{KN}$ line element can be written as (Puglies, Daniela, et al. 2013).

$$
d s^{2}=-\left(1-\frac{\left(2 \mathrm{mr}-\mathrm{Q}^{2}\right)}{\Sigma}\right) c^{2} d t^{2}-\frac{2 a\left(2 \mathrm{mr}-\mathrm{Q}^{2}\right)}{\Sigma} \sin ^{2} \theta d t d \phi+\frac{A \sin ^{2} \theta}{\Sigma} d \phi^{2}+\frac{\Sigma}{\Delta} d r^{2}+\Sigma d \theta^{2}
$$

Using geometrized units with $(G=1=c)$, and

$$
\Delta=r^{2}-2 m r+a^{2}+Q^{2}, \Sigma=r^{2}+a^{2} \cos ^{2} \theta \text { And } A=\left(r^{2}+a^{2}\right)^{2}-\Delta a^{2} \sin ^{2} \theta
$$

The parameter $a$ stands for the angular momentum per unit mass, as measured by a distant observer. The limiting case of the KN metric is the Kerr metric (Kerr. R P, 1963) for $Q=0$, The Schwarzschild metric which is recovered for $a=Q=$ 0 , the Reissner-Nordström (RN) space time for $a=0$, and the Minkowski metric for special relativity for $a=Q=m=$ 0 . The KN spacetime is asymptotically flat and free of curvature singularities outside the region situated very close to the origin of coordinates (Puglies, Daniela, et al. 2013). In particular, the function $\Delta$ vanishes at the radii

$$
r_{ \pm}=m \pm \sqrt{m^{2}-a^{2}-Q^{2}}
$$

Become real only if the condition $m^{2}>Q^{2}+a^{2}$ is satisfied. In this case $r_{+}$and $r_{-}$represents the radii of the outer and inner horizon, respectively and the $\mathrm{KN}$ solution is interpreted as describing the exterior field of a rotating black hole. In the case $m^{2}<Q^{2}+a^{2}$, no zero of $\Delta$ exits and the gravitational field correspond to that of a ring singularities situated at (Lynden- Bell. 2004).

$r^{2}+a^{2} \cos ^{2} \theta=0$, Then for positive in the region 
$r^{2}+a^{2} \cos ^{2} \theta+Q^{2}-2 m r<0$ or $\quad r_{-s}<r<r_{+_{s}} \quad$ with $r_{ \pm s}=m \pm \sqrt{m^{2}-Q^{2}-a^{2} \cos ^{2} \theta}$

In particular, for a black hole it is $r_{+}<r<r_{+_{s}}$ a region

$$
g_{t t}=-\left(1-\frac{\left(2 \mathrm{mr}-\mathrm{Q}^{2}\right)}{\Sigma}\right), g_{r r}=\frac{\Sigma}{\Delta_{k}}, g_{\theta \theta}=\Sigma, g_{\phi \phi}=\frac{A}{\Sigma} \sin ^{2} \theta, g_{t \phi}=-2 a \sin ^{2} \theta \frac{\left(2 \mathrm{mr}-\mathrm{Q}^{2}\right)}{\Sigma},
$$

$m=\frac{M G}{c^{2}}, c$ Being the velocity of light.

\section{Hydrodynamical Equation for Kerr Newman Background Geometry}

The equation governing the motion of perfect fluid is described by law of conservation of the energy-momentum tensor

$$
T_{, j}^{i j}=0
$$

Where $p$ is the pressure, $\rho$ is the energy density including rest mass energy and $u^{i}$ is the four velocity satisfying the normalization relationship $u^{i} u_{i}=1$.

Momentum equation for

$$
\left(\rho+\frac{p}{c^{2}}\right) u_{i j}^{i} ; u^{j}=\frac{1}{c^{2}}\left(g^{i j}-u^{i} u^{j}\right) p_{, j}
$$

Continuity equation

$$
\rho_{, i} u^{i}+\left(\rho+\frac{p}{c^{2}}\right) u_{i j}^{i} ;=0
$$

The equation of momentum and continuity in terms of 3- velocity $V^{\alpha}$ (Prasanna, AR, 1982 ).

$$
\begin{gathered}
\left(\rho+\frac{p}{c^{2}}\right)\left(u^{0}\right)^{2}\left[\frac{d V^{\alpha}}{d t}+c^{2}\left(\Gamma_{00}^{\alpha}-\frac{\mathrm{V}^{\alpha}}{\mathrm{c}} \Gamma_{00}^{0}\right)+2 c \mathrm{~V}^{\beta}\left(\Gamma_{0 \beta}^{\alpha}-\frac{\mathrm{V}^{\alpha}}{\mathrm{c}} \Gamma_{0 \beta}^{0}\right)+\mathrm{V}^{\gamma} \mathrm{V}^{\beta}\left(\Gamma_{\beta \gamma}^{\alpha}-\frac{\mathrm{V}^{\alpha}}{\mathrm{c}} \Gamma_{\beta \gamma}^{0}\right)\right]+\left(g^{0 i} \frac{\mathrm{V}^{\alpha}}{\mathrm{c}}-\mathrm{g}^{\alpha \mathrm{i}}\right) \frac{\partial p}{\partial x^{i}}=\left(F_{k}^{\alpha}-\right. \\
\left.F_{k}^{0} \frac{\mathrm{V}^{\alpha}}{\mathrm{c}}\right) \frac{J^{k}}{c} \\
\left(\rho+\frac{p}{c^{2}}\right)\left[V_{, \alpha}^{\alpha}+c \Gamma_{0 \alpha}^{\alpha}-\left(\Gamma_{0 \alpha}^{0}-\Gamma_{\beta \alpha}^{\beta}\right) \mathrm{V}^{\alpha}-\Gamma_{\alpha \beta}^{0} \frac{\mathrm{V}^{\alpha} \mathrm{V}^{\beta}}{\mathrm{c}}\right]+\frac{\partial}{\partial t}\left(\rho-\frac{p}{c^{2}}\right)+\mathrm{V}^{\alpha} \frac{\partial}{\partial \mathrm{x}^{\alpha}}\left(\rho-\frac{p}{c^{2}}\right)+\frac{1}{c^{2}\left(u^{0}\right)^{2}}\left(g^{00} \frac{\partial p}{\partial t}+c g^{0 \alpha} \frac{\partial p}{\partial x^{\alpha}}\right)+ \\
\frac{1}{c^{2}\left(u^{0}\right)^{2}}\left[F_{k}^{0} J^{k}-2 F_{i k} J^{k} u^{i} u^{0}\right]=0
\end{gathered}
$$

Particularizing to the Kerr Newman metric having no electromagnetic for perfect fluid disk in Kerr- Newman background geometry then equation of motion reduce into the form

$$
\begin{aligned}
& \left(\rho+\frac{p}{c^{2}}\right)\left[\frac{d V^{r}}{d t}+\left(\mathrm{V}^{\mathrm{r}} \frac{\partial \mathrm{V}^{\mathrm{r}}}{\partial \mathrm{r}}+\mathrm{V}^{\theta} \frac{\partial \mathrm{V}^{\mathrm{r}}}{\partial \theta}+\mathrm{V}^{\phi} \frac{\partial \mathrm{V}^{\mathrm{r}}}{\partial \phi}\right)\right]+c^{2} \Gamma_{t t}^{r}+2 c \mathrm{~V}^{\phi} \Gamma_{t \phi}^{r}-2 \mathrm{~V}^{\mathrm{r}}\left(\mathrm{V}^{\mathrm{r}} \Gamma_{t r}^{t}+\mathrm{V}^{\theta} \Gamma_{t \theta}^{t}\right)+ \\
& V^{r^{2}} \Gamma_{r r}^{r} 2 \mathrm{~V}^{\mathrm{r}} \mathrm{V}^{\theta} \Gamma_{r \theta}^{r}+V^{\theta^{2}} \Gamma_{\theta \theta}^{r}+V^{\phi^{2}} \Gamma_{\phi \phi}^{r}-\frac{\mathrm{V}^{\mathrm{r}}}{c}\left[2 \mathrm{~V}^{\mathrm{r}} \mathrm{V}^{\theta} \Gamma_{t \phi}^{r}-\mathrm{V}^{\theta} \mathrm{V}^{\phi \Gamma_{\theta \phi}^{t}}\right]= \\
& \frac{1}{\left(u^{0}\right)^{2}}\left[\frac{\mathrm{V}^{\mathrm{r}}}{c^{2}} \frac{A}{\Delta_{k} \Sigma} \frac{\partial p}{\partial t}-\frac{\Delta_{k}}{\Sigma} \frac{\partial p}{\partial r}-\frac{\mathrm{V}^{\mathrm{r}}}{\mathrm{c}} \frac{\mathrm{a}\left(2 \mathrm{mr}-\mathrm{Q}^{2}\right)}{\Delta_{k} \Sigma} \frac{\partial p}{\partial \phi}\right] \\
& \left(\rho+\frac{p}{c^{2}}\right)\left[\frac{d V^{\theta}}{d t}+\left(\mathrm{V}^{\mathrm{r}} \frac{\partial \mathrm{V}^{\theta}}{\partial \mathrm{r}}+\mathrm{V}^{\theta} \frac{\partial \mathrm{V}^{\theta}}{\partial \theta}+\mathrm{V}^{\phi} \frac{\partial \mathrm{V}^{\phi}}{\partial \phi}\right)+c^{2} \Gamma_{t t}^{\theta}+2 c \mathrm{~V}^{\phi}-2 \mathrm{~V}^{\theta}\left(\mathrm{V}^{\mathrm{r}} \Gamma_{r r}^{t}+\mathrm{V}^{\theta} \Gamma_{t \theta}^{t}\right)+V^{r^{2}} \Gamma_{r r}^{\theta}+2 \mathrm{~V}^{\mathrm{r}} \mathrm{V}^{\theta} \Gamma_{r \theta}^{\theta}+V^{\theta^{2}} \Gamma_{\theta \theta}^{\theta}\right. \\
& \left.+V^{\phi^{2}} \Gamma_{\phi \phi}^{\theta}-\frac{\mathrm{V}^{\theta}}{\mathrm{c}}\left[2 \mathrm{~V}^{\mathrm{r}} \mathrm{V}^{\theta} \Gamma_{r \phi}^{r}+2 \mathrm{~V}^{\theta} \mathrm{V}^{\phi} \Gamma_{\theta \phi}^{t}\right]\right] \\
& =\frac{1}{\left(u^{0}\right)^{2}}\left[-\frac{\mathrm{V}^{\theta}}{c} \frac{A}{\Delta_{k} \Sigma} \frac{\partial p}{\partial t}-\frac{1}{\Sigma} \frac{\partial p}{\partial \theta}-\frac{\mathrm{V}^{\theta}}{\mathrm{c}} \frac{\mathrm{a}\left(2 \mathrm{mr}-\mathrm{Q}^{2}\right)}{\Delta_{k} \Sigma} \frac{\partial p}{\partial \phi}\right] \\
& \left(\rho+\frac{p}{c^{2}}\right)\left[\frac{d V^{\phi}}{d t}+\left(\mathrm{V}^{\mathrm{r}} \frac{\partial \mathrm{V}^{\phi}}{\partial \mathrm{r}}+\mathrm{V}^{\theta} \frac{\partial \mathrm{V}^{\phi}}{\partial \theta}+\mathrm{V}^{\phi} \frac{\partial \mathrm{V}^{\phi}}{\partial \phi}\right)+2 \mathrm{c}\left(\mathrm{V}^{\mathrm{r}} \Gamma_{t r}^{\phi}+\mathrm{V}^{\theta} \Gamma_{t \phi}^{\phi}\right)-2 \mathrm{~V}^{\phi}\left(\mathrm{V}^{\mathrm{r}} \Gamma_{t r}^{t}+\mathrm{V}^{\theta} \Gamma_{t \theta}^{t}\right)+2 \mathrm{~V}^{\mathrm{r}} \mathrm{V}^{\phi} \Gamma_{r \phi}^{\phi}+2 \mathrm{~V}^{\theta} \mathrm{V}^{\phi}-\right. \\
& \left.\frac{\mathrm{V}^{\phi}}{\mathrm{c}}\left[2 \mathrm{~V}^{\mathrm{r}} \mathrm{V}^{\phi} \Gamma_{r \phi}^{t}+2 \mathrm{~V}^{\theta} \mathrm{V}^{\phi} \Gamma_{\theta \phi}^{t}\right]\right]=\left[\frac{\partial p}{\partial t}\left(\frac{\mathrm{a}\left(2 \mathrm{mr}-\mathrm{Q}^{2}\right)-\sin ^{2} \theta}{\Delta_{k} \Sigma}-\frac{\mathrm{V}^{\phi}}{\mathrm{c}} \frac{\mathrm{A}}{\Delta_{k} \Sigma}\right)-\left\{\left(\frac{\Sigma}{A \sin ^{2} \theta}-a^{2} \frac{\left(2 \mathrm{mr}-\mathrm{Q}^{2}\right)^{2}}{A \Delta_{k} \Sigma}\right)+\frac{\mathrm{V}^{\phi}}{\mathrm{c}} \frac{\mathrm{a}\left(2 \mathrm{mr}-\mathrm{Q}^{2}\right)}{\Delta_{k} \Sigma}\right\} \frac{\partial p}{\partial \phi} \frac{1}{\left(u^{0}\right)^{2}}\right]
\end{aligned}
$$


Continuity equation (Chakraborty D K and Prasanna A R. 1981)

$$
\begin{gathered}
\left(\rho+\frac{p}{c^{2}}\right)\left[\frac{\partial \mathrm{V}^{\mathrm{r}}}{\partial \mathrm{r}}+\frac{\partial \mathrm{V}^{\mathrm{r}}}{\partial \theta}+\frac{\partial \mathrm{V}^{\mathrm{r}}}{\partial \phi}-\mathrm{V}^{\mathrm{r}} \Gamma_{t r}^{t}-\mathrm{V}^{\theta} \Gamma_{t \phi}^{t}+\mathrm{V}^{\mathrm{r}}\left(\Gamma_{t \phi}^{\phi}+\Gamma_{r r}^{r}+\Gamma_{r \theta}^{\theta}\right)+\mathrm{V}^{\theta}\left(\Gamma_{\theta r}^{r}+\Gamma_{\theta \phi}^{\phi}+\Gamma_{\theta \theta}^{\theta}\right)-\frac{\mathrm{V}^{\mathrm{r}} \mathrm{V}^{\phi}}{\mathrm{c}} \Gamma_{r \phi}^{t}-\frac{\mathrm{V}^{\phi} \mathrm{V}^{\theta}}{\mathrm{c}} \Gamma_{\theta \phi}^{t}\right]+ \\
\left(\frac{\partial}{\partial \mathrm{t}}+\mathrm{V}^{\mathrm{r}} \frac{\partial}{\partial \mathrm{r}}+\mathrm{V}^{\theta} \frac{\partial}{\partial \theta}+\mathrm{V}^{\phi} \frac{\partial}{\partial \phi}\right)\left(\rho+\frac{p}{c^{2}}\right)+\frac{1}{c\left(u^{0}\right)^{2}}\left(\frac{A}{\Delta_{k} \Sigma} \frac{\partial p}{c \partial t}-\frac{\mathrm{a}\left(2 \mathrm{mr}-\mathrm{Q}^{2}\right)}{\Delta_{k} \Sigma} \frac{\partial p}{\partial \phi}\right)=0
\end{gathered}
$$

Adiabatic equation

$$
\left(\frac{\partial}{\partial \mathrm{t}}+\mathrm{V}^{\mathrm{r}} \frac{\partial}{\partial \mathrm{r}}+\mathrm{V}^{\theta} \frac{\partial}{\partial \theta}+\mathrm{V}^{\phi} \frac{\partial}{\partial \phi}\right) \frac{\mathrm{p}}{\mathrm{n} \gamma}=0
$$

Baryon conservation equation

$$
\begin{gathered}
n\left[\frac{\partial \mathrm{V}^{\mathrm{r}}}{\partial \mathrm{r}}+\frac{\partial \mathrm{V}^{\mathrm{r}}}{\partial \theta}+\frac{\partial \mathrm{V}^{\mathrm{r}}}{\partial \phi}-\mathrm{V}^{\mathrm{r}} \Gamma_{t r}^{t}-\mathrm{V}^{\theta} \Gamma_{t \theta}^{t}+\mathrm{V}^{\mathrm{r}}\left(\Gamma_{r \phi}^{\phi}+\Gamma_{r r}^{r}+\Gamma_{r \theta}^{\theta}\right)+\mathrm{V}^{\theta}\left(\Gamma_{\theta \phi}^{\phi}+\Gamma_{\theta r}^{r}+\Gamma_{\theta \theta}^{\theta}\right)-\frac{\mathrm{V}^{\mathrm{r}} \mathrm{V}^{\phi}}{\mathrm{c}} \Gamma_{r \phi}^{t}-\frac{\mathrm{V}^{\phi} \mathrm{V}^{\theta}}{\mathrm{c}} \Gamma_{\theta \phi}^{t}\right]+\left(\frac{\partial}{\partial \mathrm{t}}+\mathrm{V}^{\mathrm{r}} \frac{\partial}{\partial \mathrm{r}}+\right. \\
\left.\mathrm{V}^{\theta} \frac{\partial}{\partial \theta}+\mathrm{V}^{\phi} \frac{\partial}{\partial \phi}\right) \mathrm{n}+\mathrm{n}\left\{\frac{1}{c\left(u^{0}\right)^{2}}\left(\frac{A}{\Delta_{k} \Sigma} \frac{\partial p}{\partial \phi}+\frac{\mathrm{a}\left(2 \mathrm{mr}-\mathrm{Q}^{2}\right)}{\Delta_{k} \Sigma} \frac{\partial p}{\partial \phi}\right)\right\}-\frac{1}{c^{2}}\left(\frac{\partial}{\partial \mathrm{t}}+\mathrm{V}^{\mathrm{r}} \frac{\partial}{\partial \mathrm{r}}+\mathrm{V}^{\theta} \frac{\partial}{\partial \theta}+\mathrm{V}^{\phi} \frac{\partial}{\partial \phi}\right) p=0
\end{gathered}
$$

The above equation gives the completehydrodynamical equation of Kerr Newman black hole.

Calculating all nonvanishing connection coefficient components $\Gamma$ as follows

$$
\begin{aligned}
& \Gamma_{t r}^{t}=\frac{r^{2}+a^{2}}{\Delta \Sigma}\left[m\left(r^{2}-a^{2} \cos ^{2} \theta\right)-r Q^{2}\right], \Gamma_{t \theta}^{t}=\frac{a^{2} \sin \theta \cos \theta}{\Sigma^{2}}\left(r^{2}+a^{2}-\Delta\right), \\
& \Gamma_{\phi r}^{t}=-\frac{a \sin ^{2} \theta}{\Delta \Sigma^{2}}\left[\left\{m\left(r^{2}-a^{2} \cos ^{2} \theta\right)-r Q^{2}\right\}\left(r^{2}+a^{2}\right)-r \Sigma\left(r^{2}+a^{2}-\Delta\right)\right], \\
& \Gamma_{\phi \theta}^{t}=\frac{a^{3} \sin ^{3} \theta \cos \theta}{\Sigma^{2}}\left(r^{2}+a^{2}-\Delta\right), \Gamma_{t r}^{\phi}=\frac{a}{\Delta \Sigma^{2}}\left(m\left(r^{2}-a^{2} \cos ^{2} \theta\right)-r Q^{2}\right), \\
& \Gamma_{t \theta}^{\phi}=-\frac{a \cot \theta}{\Sigma^{2}}\left(r^{2}+a^{2}-\Delta\right), \Gamma_{\phi r}^{\phi}=-\frac{1}{\Delta \Sigma^{2}}\left[\left\{m\left(r^{2}-a^{2} \cos ^{2} \theta\right)-r Q^{2}\right\} a^{2} \sin ^{2} \theta-r \Sigma\left(\Delta-a^{2} \sin ^{2} \theta\right)\right] \\
& \Gamma_{\phi \theta}^{\phi}=-\frac{\cot \theta}{\Sigma^{2}}\left[\Sigma\left(\Delta-a^{2} \sin ^{2} \theta \Sigma^{2} \theta\right)+\left(r^{2}+a^{2}\right)\left(r^{2}+a^{2}-\Delta\right)\right], \\
& \Gamma_{t t}^{r}=\frac{\Delta}{\Sigma^{3}}\left[m\left(r^{2}-a^{2} \cos ^{2} \theta\right)-r Q^{2}\right], \Gamma_{t \phi}^{r}=\frac{\Delta a \sin { }^{2} \theta}{\Sigma^{3}}\left[m\left(r^{2}-a^{2} \cos ^{2} \theta\right)-r Q^{2}\right], \\
& \Gamma_{\phi \phi}^{r}=\frac{\Delta \sin { }^{2} \theta}{\Sigma^{3}}\left[r \Sigma^{2}-a^{2} \sin ^{2} \theta\left(m\left(r^{2}-a^{2} \cos { }^{2} \theta\right)-r Q^{2}\right)\right] \\
& \Gamma_{r r}^{r}=\frac{1}{\Delta \Sigma}[r(\Delta-\Sigma)+\mathrm{m} \Sigma], \Gamma_{r \theta}^{r}=-\frac{a^{2} \sin \theta \cos \theta}{\Sigma}, \Gamma_{\theta \theta}^{r}=-\frac{\Delta r}{\Sigma}, \\
& \Gamma_{t t}^{\theta}-\frac{a^{2} \sin \theta \cos \theta}{\Sigma^{2}}\left(r^{2}+a^{2}-\Delta\right), \Gamma_{t \phi}^{\theta}-\frac{a \sin \theta \cos \theta}{\Sigma^{2}}\left(r^{2}+a^{2}\right)\left(r^{2}+a^{2}-\Delta\right) \\
& \Gamma_{\phi}^{2}\left[\Delta \Sigma^{2}+\left(r^{2}+a^{2}\right)\left(r^{2}+a^{2}-\Delta\right)\right], \Gamma_{r r}^{\theta}=\frac{a^{2} \sin \theta \cos \theta}{\Delta \Sigma}, \Gamma_{r \theta}^{\theta}=\frac{r}{\Sigma}, \Gamma_{\theta \theta}^{\theta}=-\frac{a^{2} \sin _{\theta} \cos \theta}{\Sigma}
\end{aligned}
$$

\section{Dynamics of the Disk with Radial Flow in Locally Non-rotating Frame (L. N. R. F)}

In LNRF Physical phenomena becomesmore transparent because it cancels out the effect of frame dragging of black hole rotation as observeris chosen to rotate with the black hole. The observer whose world line are $r=\operatorname{constant}, \theta=\operatorname{constant}$ and $\phi=\omega t+$ constant Where $\omega=-\frac{g_{t \phi}}{g_{\phi \phi}}$, although these observer are rotating, the geometryisflat for the observer (Bardeen. $\mathrm{J}$ M, 1973).

Velocity $V^{\alpha}$ in LNRF expressed as

$$
V^{\alpha}=\frac{\lambda_{\beta}^{(\alpha)} V^{\beta}+\lambda_{t}^{(\alpha)}}{\lambda_{\beta}^{(t)} V^{\beta}+\lambda_{\beta}^{(t)}}
$$

With this geometry, introducing a locally nonrotating frame (Kinnersley. W, 1969). 


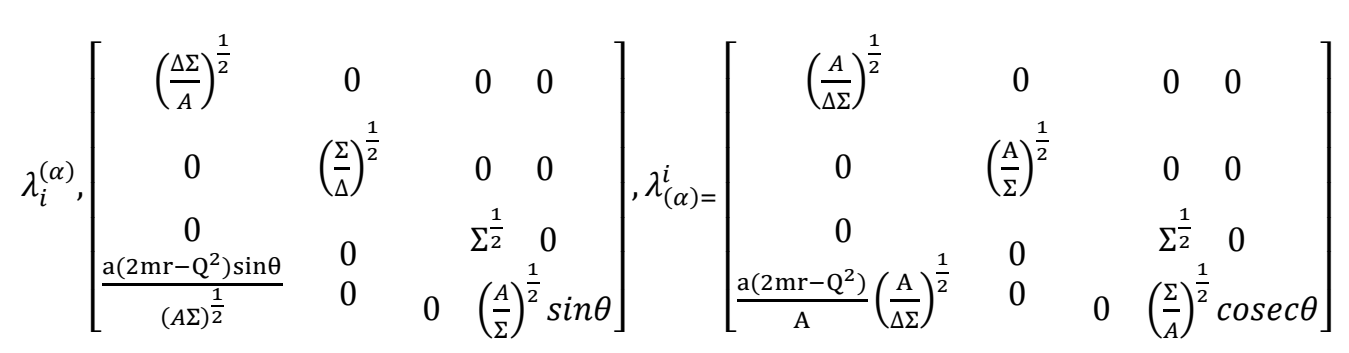

Now restricting to the case of slowly rotating Kerr new man black hole (Newman, E T. et al. 1965) (a « 1). In principle, the form of Kerr Newman background for the equation of motion written in terms of $V^{\alpha}$ The time components $u^{0}$ of the four velocity which appear in the momentum equations expressed as

$$
\begin{gathered}
\frac{1}{\left(u^{0}\right)^{2}}=\left(1-\frac{\left(2 \mathrm{mr}-\mathrm{Q}^{2}\right)}{\Sigma}\right)+2 \operatorname{asin}^{2} \theta \frac{\left(2 \mathrm{mr}-\mathrm{Q}^{2}\right)}{\Sigma} \frac{\mathrm{v}^{\phi}}{\mathrm{c}}-\frac{\Sigma}{\Delta_{k}}\left(\frac{\mathrm{v}^{\mathrm{r}}}{\mathrm{c}}\right)^{2}-\Sigma\left(\frac{\mathrm{V}^{\theta}}{\mathrm{c}}\right)^{2}-\frac{A}{\Sigma} \sin ^{2}\left(\frac{\mathrm{V}^{\phi}}{\mathrm{c}}\right)^{2} \\
\frac{1}{\left(u^{0}\right)^{2}}=\left(1-\frac{\left(2 \mathrm{mr}-\mathrm{Q}^{2}\right)}{\Sigma}\right)\left(1-\frac{V^{2}}{c^{2}}\right)
\end{gathered}
$$

Where $V^{2}=\mathrm{V}^{(\mathrm{r})^{2}}+\mathrm{V}^{(\theta)^{2}}+\mathrm{V}^{(\phi)^{2}}$

$$
\begin{aligned}
& \frac{\mathrm{V}^{\mathrm{r}}}{\mathrm{c}}=\left(1-\frac{\left(2 \mathrm{mr}-\mathrm{Q}^{2}\right)}{\Sigma}\right)^{1 / 2} \\
& \frac{\mathrm{v}^{\theta}}{\mathrm{c}}=\frac{1}{\mathrm{r}}\left(1-\frac{\left(2 \mathrm{mr}-\mathrm{Q}^{2}\right)}{\Sigma}\right)^{1 / 2} \mathrm{~V}^{(\theta)} \\
& \frac{\mathrm{v}^{\phi}}{\mathrm{c}}=\frac{1}{\mathrm{r} \sin \theta}\left(1-\frac{\left(2 \mathrm{mr}-\mathrm{Q}^{2}\right)}{\Sigma}\right)^{1 / 2} \mathrm{~V}^{(\phi)}+\frac{\mathrm{ac}\left(2 \mathrm{mr}-\mathrm{Q}^{2}\right)}{\Sigma^{2}}
\end{aligned}
$$

Limiting for thin disk, $\mathrm{V}^{(\theta)}=0$ thus the equations for radial flow reduces to stationary state with axis symmetric as follows

$$
\begin{gathered}
\left(\rho+\frac{p}{c^{2}}\right)=\left[\left(1-\frac{\left(2 \mathrm{mr}-\mathrm{Q}^{2}\right)}{\Sigma}\right) \mathrm{V}^{(r)} \frac{\partial \mathrm{V}^{(r)}}{\partial \mathrm{r}}-\frac{1}{\mathrm{r}}\left(1-\frac{\left(2 \mathrm{mr}-\mathrm{Q}^{2}\right)}{\Sigma}\right) \mathrm{V}^{(\phi)^{2}}-\frac{6 a m c}{r^{3}}\left(1-\frac{\mathrm{V}^{(\mathrm{r})^{2}}}{c^{2}}\right)\left(1-\frac{\left(2 \mathrm{mr}-\mathrm{Q}^{2}\right)}{\Sigma}\right)^{1 / 2} \sin \theta \mathrm{V}^{(\phi)}\right]= \\
-\left(1-\frac{\left(2 \mathrm{mr}-\mathrm{Q}^{2}\right)}{\Sigma}\right)\left(1-\frac{\mathrm{V}^{2}}{c^{2}}\right) \frac{\partial p}{\partial r} \\
\left(\rho+\frac{p}{c^{2}}\right)=\left[-\frac{\cot \theta}{r}\left(1-\frac{\left(2 \mathrm{mr}-\mathrm{Q}^{2}\right)}{\Sigma}\right)^{1 / 2} \mathrm{~V}^{(\phi)^{2}}\right]=-\left(1-\frac{\mathrm{V}^{2}}{c^{2}}\right) \frac{1}{r}\left(1-\frac{\left(2 \mathrm{mr}-\mathrm{Q}^{2}\right)}{\Sigma}\right)^{1 / 2} \frac{\partial p}{\partial \theta} \\
\frac{\partial}{\partial r}\left(\log \mathrm{V}^{(\phi)}\right)+\frac{1}{r}\left(1-\frac{\left(2 \mathrm{mr}-\mathrm{Q}^{2}\right)}{\Sigma}\right)^{-1}\left(1-\frac{\left(3 \mathrm{mr}-\mathrm{Q}^{2}\right)}{\Sigma}\right)+\frac{6 a m c}{r^{3}} \sin \theta\left(1-\frac{\left(2 \mathrm{mr}-\mathrm{Q}^{2}\right)}{\Sigma}\right)^{1 / 2} \mathrm{~V}^{(\phi)}=0 \\
\left(\rho+\frac{p}{c^{2}}\right)=\left[\left(1-\frac{\left(2 \mathrm{mr}-\mathrm{Q}^{2}\right)}{\Sigma}\right) \frac{1}{r^{2}} \frac{\partial}{\partial r}\left(r^{2} \mathrm{~V}^{(r)}\right)+\frac{6 a m c}{r^{3}} \sin \theta \mathrm{V}^{(r)} \mathrm{V}^{(\phi)}\right]+\left(1-\frac{\left(2 \mathrm{mr}-\mathrm{Q}^{2}\right)}{\Sigma}\right) \mathrm{V}^{(r)} \frac{\partial}{\partial \mathrm{r}}\left(\rho+\frac{p}{c^{2}}\right)=0 \\
n\left[\left(1-\frac{\left(2 \mathrm{mr}-\mathrm{Q}^{2}\right)}{\Sigma}\right) \frac{1}{r^{2}} \frac{\partial}{\partial r}\left(r^{2} \mathrm{~V}^{(r)}\right)+\frac{6 a m c}{r^{3}} \sin \theta \mathrm{V}^{(r) \mathrm{V}^{(\phi)}}\right]+\left(1-\frac{\left(2 \mathrm{mr}-\mathrm{Q}^{2}\right)}{\Sigma}\right) \mathrm{V}^{(r)} \frac{\partial \mathrm{n}}{\partial \mathrm{r}}+\frac{\mathrm{n}}{\left(\rho+\frac{p}{c^{2}}\right)}\left\{-\frac{1}{c^{2}}\left(1-\frac{\left(2 \mathrm{mr}-\mathrm{Q}^{2}\right)}{\Sigma}\right) \mathrm{V}^{(r)} \frac{\partial \mathrm{p}}{\partial \mathrm{r}}\right\}=0(28)
\end{gathered}
$$

and

$$
p \frac{\partial}{\partial t}\left(\frac{1}{\mathrm{n}^{\gamma}}\right)+\left(1-\frac{\left(2 \mathrm{mr}-\mathrm{Q}^{2}\right)}{\Sigma}\right) \mathrm{V}^{(r)} \frac{\partial}{\partial \mathrm{r}}\left(\frac{\mathrm{p}}{\mathrm{n}^{\gamma}}\right)=0
$$

Where $\gamma=\frac{c_{p}}{c_{v}}$, The law of thermodynamics which includes the law of concentration of baryon numbers.

$$
\left(n u^{i}\right) ; j=0
$$

Where $n$ is number density baryon numbers. In case of pressureless dust $p=0$ and $\theta=\frac{\pi}{2}$ the fluid disk confine to the equatorial plane the baryon conservation equation together with continuity equation can be written as (Bhaskaran, P and Prasanna, A R. 1990).

$$
n \frac{\partial \rho}{\partial n}=\left(\rho+\frac{p}{c^{2}}\right) \frac{\partial n}{\partial r} 0
$$

While the adiabatic equation of state is written as 


$$
n \frac{\partial p}{\partial r}=r p \frac{\partial n}{\partial r}
$$

\section{Thin Disk}

Confining to the case of cold disk with $p=0$ and $\theta=\frac{\pi}{2}$ for negligible pressure the adiabatic equation and baryon conservation equation become identically zero and left with equation three only (Mishra, K N. and Chakraborty. D K, 1999). Rewriting equations in dimensionless form,

$$
\begin{gathered}
\left(1-\frac{\left(2 \mathrm{mr}-\mathrm{Q}^{2}\right)}{\Sigma}\right) \frac{\partial \mathrm{V}^{(\phi)}}{\partial r}+\left(1-\frac{\left(3 \mathrm{mr}-\mathrm{Q}^{2}\right)}{\Sigma}\right) \frac{\mathrm{V}^{(\phi)}}{r}+\frac{6 a m c \sin \theta}{r^{3}}\left(1-\frac{\left(2 \mathrm{mr}-\mathrm{Q}^{2}\right)}{\Sigma}\right)^{1 / 2} \mathrm{~V}^{(\phi)}=0 \\
\left(1-\frac{\left(2 \mathrm{mr}-\mathrm{Q}^{2}\right)}{\Sigma}\right) \frac{\partial \mathrm{V}^{(\phi)}}{\partial r}-\left(1-\frac{\left(3 \mathrm{mr}-\mathrm{Q}^{2}\right)}{\Sigma}\right) \frac{\mathrm{v}^{\mathrm{r}}}{r} \mathrm{~V}^{\mathrm{r}}+\frac{6 a m \sin \theta}{r^{3}}\left(\mathrm{~V}^{(r)^{2}}-1\right) \mathrm{V}^{(\phi)}\left(1-\frac{\left(2 \mathrm{mr}-\mathrm{Q}^{2}\right)}{\Sigma}\right)^{1 / 2}=0
\end{gathered}
$$

and

$$
\begin{gathered}
\rho\left[\left(1-\frac{\left(2 \mathrm{mr}-\mathrm{Q}^{2}\right)}{\Sigma}\right) \frac{1}{r^{2}} \frac{\partial}{\partial r}\left(r^{2} \mathrm{~V}^{(r)}\right)+\frac{6 a m \sin \theta}{r^{3}}\left(1-\frac{\left(2 \mathrm{mr}-\mathrm{Q}^{2}\right)}{\Sigma}\right)^{1 / 2} \mathrm{~V}^{(r)} \mathrm{V}^{(\phi)}\right]+\left(1-\frac{\left(2 \mathrm{mr}-\mathrm{Q}^{2}\right)}{\Sigma}\right) \mathrm{V}^{(r)} \frac{\partial \rho}{\partial r}=0 \\
\mathrm{~V}^{(\phi)}=\frac{\mathrm{B}}{\mathrm{r}}\left(1-\frac{\left(2 \mathrm{mr}-\mathrm{Q}^{2}\right)}{\Sigma}\right)^{1 / 2}\left(1+B \frac{a \sin \theta}{r} \frac{\left(2 \mathrm{mr}-\mathrm{Q}^{2}\right)}{\Sigma}\right) \\
\mathrm{V}^{(\mathrm{r})^{2}}=1-\left[\left(1-\frac{\left(2 \mathrm{mr}-\mathrm{Q}^{2}\right)}{\Sigma}\right)\left\{1+\frac{B^{2}}{2} \frac{\left(2 \mathrm{mr}-\mathrm{Q}^{2}\right)}{\Sigma}\right\}\left(1+B \frac{2 a \sin \theta}{r} \frac{\left(2 \mathrm{mr}-\mathrm{Q}^{2}\right)}{\Sigma}\right)\right] \\
\rho=\frac{\rho_{c}}{r^{2} \mathrm{~V}^{(r)}}\left(1+B \frac{a \sin \theta}{r} \frac{\left(2 \mathrm{mr}-\mathrm{Q}^{2}\right)}{\Sigma}\right)
\end{gathered}
$$

$B$, Constant At $\theta=\frac{\pi}{2}, \Sigma=r^{2}+a^{2} \cos ^{2} \theta$ become $\Sigma=r^{2}$ and $\frac{\mathrm{Q}^{2}}{\Sigma}=$ effective potential $V=\frac{Q}{r}$ writing the expression in dimensionless form as, $Q=\frac{Q}{m} R=\frac{r}{m}, \alpha=\frac{a}{m}, m=\frac{M G}{c^{2}}$ and $V_{K N}=\frac{Q / m}{r / m}$ considered $G=c=1$.

$$
\begin{aligned}
& \mathrm{V}^{(\phi)}=\frac{\mathrm{B}}{\mathrm{R}}\left\{1-\left(\frac{2}{\mathrm{R}}-{V_{K N}}^{2}\right)\right\}^{1 / 2}\left\{1+B \frac{\alpha \sin \theta}{R}\left(\frac{2}{\mathrm{R}}-V_{K N}^{2}\right)\right\} \\
& \rho=\frac{\rho_{c}}{R^{2} \mathrm{~V}^{(r)}}\left\{1+B \frac{\alpha \sin \theta}{R}\left(\frac{2}{\mathrm{R}}-V_{K N}^{2}\right)\right\} \\
& \mathrm{V}^{(\mathrm{r})^{2}}=1-\left[\left\{1-\left(\frac{2}{\mathrm{R}}-{V_{K N}}^{2}\right)\right\}\left\{1+\frac{1}{2} B^{2}\left(\frac{2}{\mathrm{R}}-V_{K N}{ }^{2}\right)\right\}\left\{1+B \frac{2 \alpha \sin \theta}{R}\left(\frac{2}{\mathrm{R}}-V_{K N}^{2}\right)\right\}\right]
\end{aligned}
$$

At $=\frac{\pi}{2}$

$$
\begin{aligned}
& \mathrm{V}^{(\mathrm{r})^{2}}=1-\left[\left\{1-\left(\frac{2}{\mathrm{R}}-{V_{K N}}^{2}\right)\right\}\left\{1+\frac{1}{2} B^{2}\left(\frac{2}{\mathrm{R}}-{V_{K N}}^{2}\right)\right\}\left\{1+\frac{2 \alpha B}{R}\left(\frac{2}{\mathrm{R}}-V_{K N}^{2}\right)\right\}\right] \\
& \mathrm{V}^{(\phi)}=\frac{\mathrm{B}}{\mathrm{R}}\left\{1-\left(\frac{2}{\mathrm{R}}-{V_{K N}}^{2}\right)\right\}^{1 / 2}\left\{1+\frac{\alpha B}{R}\left(\frac{2}{\mathrm{R}}-V_{K N}^{2}\right)\right\} \\
& \rho=\frac{\rho_{c}}{R^{2} \mathrm{~V}^{(r)}}\left\{1+\frac{\alpha B}{R}\left(\frac{2}{\mathrm{R}}-{V_{K N}}^{2}\right)\right\}
\end{aligned}
$$

In the above $B$ and $\rho_{c}$ are two constants, for boundary condition $\mathrm{V}^{(\mathrm{r})}=0$ at $r=\infty$ and $p=0$ at $\theta=\frac{\pi}{2}$ plane.

\section{Conclusion}

The solutions $\rho, \mathrm{V}^{(\mathrm{r})^{2}}$ and $\mathrm{V}^{(\phi)}$ carry the signature of the rotation of the charge black hole, the profile for $\mathrm{V}^{(\mathrm{r})}, \mathrm{V}^{(\phi)}$ and $\rho$ for $\alpha=-0.4 .-0.2,0 .+0.2$, and +0.4 the velocity $\mathrm{V}^{(\mathrm{r})}$ decreases (increases) when $a$ is positive (negative), The density $\rho$ increase (decrease) while velocity $\mathrm{V}^{(\phi)}$ decrease (increase) for prograde (retrograde) motions as compared in the Schwarzchild case. The motion of the disk increase (decrease) for prograde (retrograde) depending on radial distance $R$. In Kerr geometry the solution show the effect of the rotation of the black hole through the term $\alpha$, as changing $\alpha$ to $-\alpha$ disks move from co rotating to counter rotating (Mishra, K N. and Chakraborty, D K. 1999 ). In the special case of Schwarzschild geometry.

$$
\begin{gathered}
\mathrm{V}^{(\phi)}=\frac{\mathrm{Bm}}{\mathrm{r}}\left(1-\frac{2 \mathrm{~m}}{\mathrm{r}}\right)^{1 / 2} \\
\mathrm{~V}^{(\mathrm{r})^{2}}=\frac{2 \mathrm{~m}}{\mathrm{r}}-\frac{\mathrm{B}^{2} \mathrm{~m}^{2}}{\mathrm{r}^{2}}\left(1-\frac{2 \mathrm{~m}}{\mathrm{r}}\right)
\end{gathered}
$$




$$
\rho=\frac{\rho_{c}}{\mathrm{~V}^{(r)}} \frac{\mathrm{m}^{2}}{\mathrm{r}^{2}}
$$

Squaring and adding (6.1) and (6.2) $\frac{1}{2}\left(\mathrm{~V}^{(\mathrm{r})^{2}}+\mathrm{V}^{(\phi)^{2}}\right)=\frac{1}{\mathrm{R}}$ obtained. In the Newtonian gravitational case the potential energy per unit mass is $-\frac{1}{R}$ and therefore the total energy per unit mass become sum of kinetic energy and potential energy i.e. total energy of the fluid to be zero at infinity. The form of $V^{(r)^{2}}$ and $V^{(\phi)^{2}}$ consistent with the law of conservation of energy in their Newtonian limits.

\section{References}

[1] Bardeen, J MandPress, W H. 1973, J, Math. Phys. 14, 7.

[2] Bhaskaran, PandPrasanna, A R. 1990 J. Astrophys. Astron. 11 49.
[3] Chakraborty D K and Prasanna A R. 1981 J. Astrophys. Astron. 2, 421.

[4] Kerr. R P. 1963. PhyRev let 11, 237.

[5] Kinnersley. W J. 1969Math. phys. 10.

[6] Lynden- Bell. 2004D. Phys. Rev. D70.

[7] Mishra, K N. and Chakraborty, D K. 1999 Astrophys. and Space sci, 260.

[8] Misner, C W. Thorne K S, and Wheeler, J A. 1972 Gravitation (Freeman and Co.).

[9] Newman, E T. et al. 1965, J. Math. Phys. 6,916.

[10] Puglies, Daniela, et al. Physical Review D, 2013. 